\title{
Modelado hidrológico de un páramo andino venezolano con afloramientos rocosos usando TOPMODEL
}

\section{Hydrologic modeling of a Venezuelan Andean páramo with rocky outcrops using TOPMODEL}

\author{
Marilena Yeguez $^{1,2}$ iD, Magdiel Ablan ${ }^{1}$ (D), Wouter Buytaert ${ }^{3}$ (D), Dimas Acevedo ${ }^{4}$, Mayanín E. \\ Rodríguez, ${ }^{4}$, Daniel Machado ${ }^{4}$ \\ ${ }^{1}$ Centro de Simulación y Modelos (CESIMO), Universidad de los Andes, Mérida, Venezuela. \\ ${ }^{2}$ Laboratorio de Prototipos, Universidad Nacional Experimental del Táchira, San Cristóbal, Venezuela. \\ 3 Ingeniería Civil y Ambiental, Imperial College London, Londres, Reino Unido. \\ ${ }^{4}$ Instituto de Ciencias Ambientales y Ecológicas (ICAE), Universidad de los Andes, Mérida, Venezuela. \\ Autor para correspondencia: mariyeg@gmail.com \\ Fecha de recepción: 20 de junio de 2019 - Fecha de aceptación: 8 de octubre de 2019
}

\section{RESUMEN}

Los afloramientos rocosos en el páramo andino venezolano constituyen una unidad geomorfológica con un área considerable. Los procesos hidrológicos de esta área fuente no son representados por TOPMODEL. Por lo tanto, este estudio tuvo por objetivo mejorar la simulación hidrológica de TOPMODEL, específicamente la descripción del flujo superficial, mediante la incorporación de flujo de este tipo generado por el área fuente rocosa. Basado en el análisis de varias investigaciones, un parámetro $a$ fue introducido para redireccionar a flujo superficial una fracción de la precipitación que cae sobre los afloramientos rocosos. Este parámetro fue calibrado, lo que condujo a una mejora de la eficiencia Nash-Sutcliffe en las fases de calibración y validación del modelo. Asumiendo un coeficiente de escorrentía de 1 en los afloramientos y de acuerdo con la calibración, la lluvia que cae en un $25 \%$ del área de afloramientos es redireccionada, posiblemente se trate del conjunto de afloramientos ubicados a lo sumo a $44 \mathrm{~m}$ de la red de drenaje. Sin embargo, otras interpretaciones son posibles, lo que requiere más investigación.

Palabras clave: TOPMODEL, modelado de cuenca, páramo andino, afloramientos rocosos.

\begin{abstract}
The rocky outcrops in the Venezuelan Andean páramo constitute a geomorphological unit with a considerable area. The hydrologic processes of this source area are not represented by the TOPMODEL. This study aimed to improve the hydrological simulation of the TOPMODEL, specifically the description of overland flow, by incorporating flow of this type generated by the rocky source area. Based on the analysis of various researches a parameter a was introduced redirecting to overland flow a fraction of the precipitation falling on the rocky outcrops. This parameter was calibrated, what led to an improvement in Nash-Sutcliffe efficiency in the model calibration and validation phase. Assuming a runoff coefficient of 1 in the outcrops and according to the calibration, the rainfall that falls on $25 \%$ of the outcrop areas is redirected, possibly it is the set of outcrops located at most $44 \mathrm{~m}$ from the drainage network. However, other interpretations are possible, what requires further research.
\end{abstract} Keywords: TOPMODEL, catchment modeling, Andean páramo, rocky outcrops.

\section{INTRODUCCIÓN}

Los estudios hidrológicos del páramo han sido desarrollados principalmente en Ecuador y Colombia. Estos páramos presentan marcadas diferencias en sus componentes biofísicos respecto a los páramos venezolanos que se caracterizan por ser páramos climáticamente estacionales y secos; con relieves abruptos y porcentajes importantes de afloramientos rocosos; con suelos jóvenes, graníticos, arenosos, poco profundos, sin estructura y de baja retención de agua; con un sistema en rosario de humedales y lagunas con alta conectividad hídrica. Por tal razón, existe un vacío en el conocimiento hidrológico de los páramos venezolanos que no puede suplirse de forma directa con los estudios desarrollados en Colombia y Ecuador (Rodríguez, 2010). Una de las características distintivas de los páramos venezolanos es la presencia de afloramientos rocosos, conocidos como parte del lecho rocoso o de otro estrato que sobresale por encima de los sedimentos o del suelo que lo recubren (OMM \& UNESCO, 2012).

Una descripción de la respuesta de los afloramientos rocosos a la lluvia y los diferentes factores que la controlan es presentada por Woo (2012), si la superficie 
de la roca es impermeable y sin almacenamiento en la superficie, y la evaporación es insignificante, se puede producir un coeficiente de escorrentía de 1.0. Esta relación rara vez se alcanza por varias razones. El aumento del ancho de las fracturas aumenta la infiltración y reduce la escorrentía superficial. La disponibilidad de almacenamiento en la superficie de la roca retiene un poco de agua de lluvia. Incluso después de que se libere el exceso de agua, se satisfacen los requisitos de almacenamiento del afloramiento cuesta abajo antes de que pueda continuar la escorrentía. La disminución de la intensidad de la lluvia y el aumento de la duración del evento ofrecen más tiempo para la infiltración y para la evaporación. Las altas tasas de evaporación son efectivas para eliminar la película de agua producida por la lluvia en la superficie de la roca y para agotar el agua contenida en las depresiones de la superficie. Estos factores no son necesariamente independientes entre sí.

A una escala de cuenca, la escorrentía de los afloramientos forma parte de la escorrentía directa o componente de escorrentía rápida (junto con la precipitación directa en el canal y el flujo superficial desde zonas selladas y saturadas) contribuyendo en más de un $41 \%$ a la escorrentía total, durante condiciones de cuenca húmeda (Suarez, Okello, Wenninger, \& Uhlenbrook, 2015). Un estudio más revelador es el de Burns et al. (2001) donde la escorrentía de un afloramiento que ocupa cerca de un tercio de las 10 ha de una cuenca en Georgia, dominó la escorrentía de caudal en condiciones de flujo máximo durante dos eventos de precipitación en el invierno de 1996. Este resultado difiere de los reportados en la mayoría de otros estudios, en cuencas forestales pequeñas, en las cuales el agua antigua o el agua subterránea dominan el flujo pico.

Se han modelado con TOPMODEL pequeñas cuencas caracterizadas por la presencia de afloramientos rocosos, obteniéndose ajustes buenos y aceptables respectivamente (Durand, Robson, \& Neal, 1992; Seibert, Bishop, \& Nyberg, 1997). Pero el comportamiento hidrológico de los afloramientos no fue modelado, para empezar el modelo usado es incapaz de distinguir dichas unidades geomorfológicas. Tampoco se reportó información sobre la extensión, ubicación u otras características de los mismos.

Un modelo que sí representa el comportamiento hidrológico de los afloramientos rocosos es el descrito y probado por Tarolli, Borga, \& Fontana (2008) se trata de un modelo de deslizamiento superficial, que utiliza un índice de humedad cuasi dinámico para predecir la distribución espacial de la saturación del suelo en respuesta a una lluvia de duración específica. Como las partes superiores del paisaje están caracterizadas por afloramientos rocosos, se toma en cuenta las diferentes velocidades de propagación de la escorrentía a través de la roca desnuda y los elementos topográficos del suelo. El modelo se aplica en una cuenca en los Alpes italianos $\left(1.47 \mathrm{~km}^{2}\right)$, con afloramientos de roca de un $28 \%$. Se determina que el modelo ofrece una mejora considerable con respecto al modelo cuasi dinámico no generalizado y el modelo de estado estacionario para predecir deslizamientos de tierra.

Lanni, McDonnell, \& Rigon (2011) fomentan el uso de su índice topográfico dinámico suavizado para describir el componente de flujo subsuperficial lateral en modelos de generación de deslizamientos de tierra y modelos conceptuales de lluvia-escorrentía; en contraposición a los índices topográficos derivados de la suposición de estado estacionario (especialmente cuando hay disponibles modelos de elevación digital de alta resolución).

Según Wilson \& Gallant (2000) la idoneidad de estos índices como herramientas de aplicación general aún tiene que ser demostrada. Por lo tanto, existe la necesidad de seguir buscando representaciones apropiadas, modelos que simulen la escorrentía y cuantifiquen el efecto de los afloramientos rocosos. Los modelos en general son una herramienta útil para probar hipótesis sobre un sistema, inclusive en sistemas hidrológico (Buytaert \& Beven, 2011). En la actualidad, una variedad de modelos hidrológicos, están siendo usados por la comunidad científica. Uno de estos modelos, TOPMODEL, concebido para pronóstico hidrológico en zonas templadas húmedas, intenta combinar los importantes efectos distribuidos de la topología de la red de canales y áreas de contribución dinámica con las ventajas de los modelos de cuenca simples con parámetros agregados (Beven \& Kirkby, 1979).

TOPMODEL se ha usado para un primer intento de modelización en el valle principal de una microcuenca de páramo venezolano (Yeguez, Ablan, Acevedo, Rodríguez, \& Machado, 2018); por ser un modelo hidrológico de base física (Durand et al., 1992), adecuado para modelar el proceso de lluvia-escorrentía en cuencas con suelo poco profundo y topografía moderada (Devi, Ganasri, \& Dwarakish, 2015), que fue usado satisfactoriamente en el páramo ecuatoriano y colombiano (Buytaert \& Beven, 2011; Gil \& Tobón, 2016). Si bien dicho valle se caracteriza por una marcada estacionalidad, los resultados obtenidos de la implementación de TOPMODEL no son del todo inaceptables, por lo que una mejor representación de los picos del hidrograma y un desplazamiento vertical de la curva de recesión pudieran hacer coincidir el hidrograma simulado con el observado.

Una manera de mejorar dichos resultados podría ser la representación de características de la cuenca que el modelo no considera, como es el caso de los afloramientos rocosos, que parecen influir de manera importante en la respuesta de la cuenca. Por lo tanto, TOPMODEL debe adaptarse para una adecuada simulación del proceso de lluvia-escorrentía en páramos con afloramientos rocosos. Afortunadamente, un número suficiente de desarrollos y cambios en los principios básicos del TOPMODEL original que han sido hechos, permiten describirlo como un "enfoque" en lugar de un modelo específico (Seibert $e t$ al., 1997).

Si bien se están obteniendo buenos resultados en la aplicación de índices cuasi dinámicos y dinámicos, la verificación de campo ha mostrado que en el páramo el índice topográfico de TOPMODEL puede identificar las áreas saturadas. Este es el resultado de los gradientes de pendiente claros y los suelos poco profundos de las cuencas (Buytaert \& Beven, 2009). Ya que estas características se presentan en el área de estudio, y dada la versatilidad del modelo TOPMODEL, esta investigación se propone modificarlo para incluir la representación de afloramientos rocosos, constituyéndose en un intento por modelar explícitamente la hidrología de estas unidades geomorfológicas en cuencas de páramo andino, unidades que también están presentes en el páramo ecuatoriano (Buytaert, Iñiguez, \& De Bièvre, 2007; Buytaert \& Beven, 2009; Buytaert \& Beven, 2011). 
2.

\section{MATERIALES Y MÉTODOS}

En este apartado se presenta una breve descripción del área de estudio, de la estructura básica y principales flujos de TOPMODEL y de la modificación llevada a cabo. También se abordan otros aspectos, como los datos que estuvieron disponibles para el modelado y el preprocesamiento que estos requirieron. Finalmente, se describen las etapas del proceso de modelado que fueron cubiertas como parte de esta investigación.

\section{1. Área de estudio}

El área de estudio es el valle principal de la microcuenca de la quebrada de Miguaguó, sector Mixteque en Mérida, Venezuela, superficie de $3.62 \mathrm{~km}^{2}$ sobre los $3600 \mathrm{~m}$ de altitud. Las precipitaciones allí presentan un régimen unimodal, con una marcada estacionalidad de dos a tres meses secos. El promedio anual de precipitación es 1170 $\pm 125 \mathrm{~mm}$. Los eventos de precipitación son en promedio de $7 \mathrm{~mm}$ solamente (Rodríguez, Acevedo, Buytaert, Ablan, \& De Bièvre, 2014), se caracterizan por ser de baja magnitud, poca intensidad y alta frecuencia (Rodríguez, 2010). Por otra parte, la radiación solar es fuerte $1200 \mathrm{~W}$ $\mathrm{m}^{-2}$, especialmente durante la estación seca (Córdova, 2014).

Las formas de relieve dominantes son los till sobre laderas, unidad que abarca el 53\% del área, seguido luego por los afloramientos rocosos (18\%), till sobre el fondo de valle $(17 \%)$ y lagunas y humedales sobre cubetas de sobre excavamiento (8\%). El resto del área lo representan morrenas de retroceso y derrubios rocosos (Rodríguez et al., 2014). Pero, según Córdova (2014) la unidad geomorfológica dominante es la ladera con manto coluvial (45\%), seguido por afloramiento rocoso $(20.2 \%)$. Según ambos autores y a pesar de las diferencias en los porcentajes, los afloramientos rocosos ocupan un segundo lugar. En la Figura 1 se muestran las distintas unidades geomorfológicas presentes en el área de estudio y seis clases de gradiente de la pendiente. Siendo la clase 30$60 \%$, escarpado según la FAO (2009), la de mayor ocupación espacial (37\% de la superficie total).

En los suelos hay predominancia de una alta fracción esquelética o gruesa sobre la fracción fina. En la fracción fina es la arena la que predomina, lo cual resulta en texturas gruesas (Franco arenoso y areno francoso). Además, los suelos en su horizonte superficial presentan alto contenido de materia orgánica de un 8 hasta $28 \%$, estrechamente ligado a las pendientes; las pendientes menores a $5 \%$ tienen los mayores valores de materia orgánica (suelos de orden Histosol y suelos minerales del orden Inceptisol y algunos con características superficiales hísticas), y a medida que aumentan las pendientes disminuye los contenidos de materia orgánica (suelos minerales jóvenes, Entisoles e Inceptisoles) (Córdova, 2014).

Toda el área de estudio posee vegetación natural. La vegetación está conformada por arbustales-rosetales bajos, arbustales altos, humedales y bosques altoandinos. El tipo de vegetación dominante es el arbustal-rosetal bajo, el cual se distribuye uniformemente sobre toda el área. Las otras formaciones vegetales se disponen en parches, inmersas dentro de la vegetación anterior. La vegetación en la ladera derecha es predominantemente a)

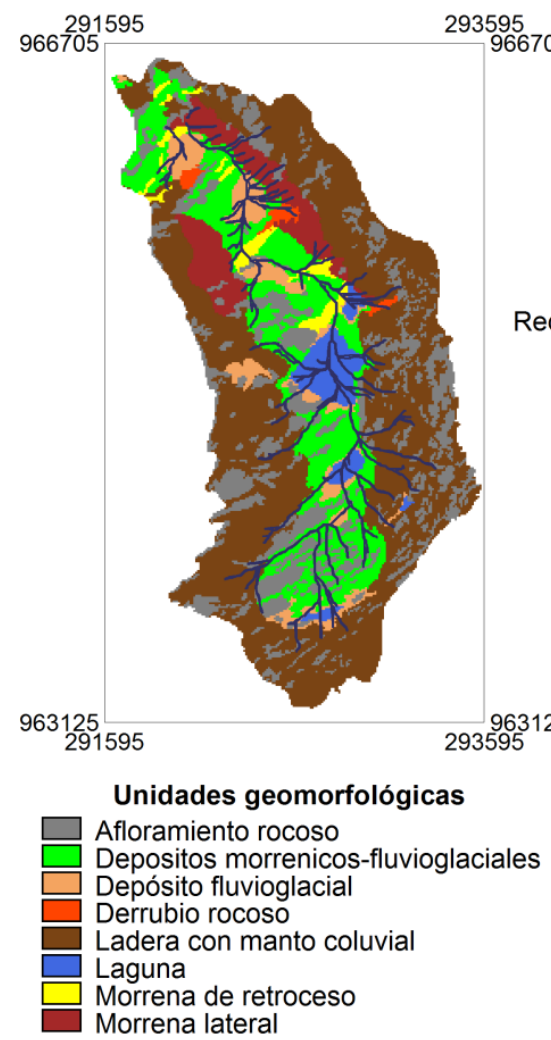

b)

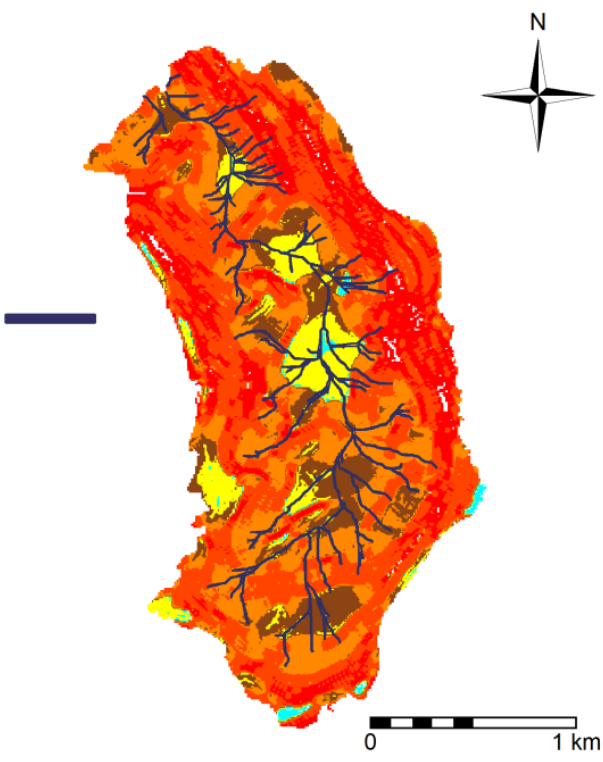

Gradiente de la pendiente

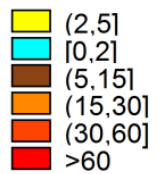

Figura 1. a) Unidades geomorfológicas; b) Gradiente de la pendiente en el área de estudio. Fuente: Córdova (2014) ajustado. 
un arbustal-rosetal con un dosel espeso. Por otra parte, los fondos de valle y la ladera izquierda están cubiertos por un arbustal de dosel abierto con mayores porciones de suelo desnudo. Mientras que los humedales están dominados por vegetación de céspedes y ciénagas (Rodríguez, 2010), conformando estos un sistema en rosario junto a las lagunas (Rodríguez et al., 2014).

\subsection{TOPMODEL}

Se usó una implementación en R de TOPMODEL, basada en la versión FORTRAN de Keith Beven de 1995 (Buytaert, 2009), cuyo código se encuentra disponible en la plataforma GITHUB (https://github.com). Su estructura y los flujos más importantes son ilustrados en la Figura 2a. En primer lugar, se calcula el flujo para infiltración, $f$, a partir de la precipitación y usando el modelo Green y Ampt (1911). Dicho flujo ingresa al suelo por medio de un primer compartimiento denominado zona radicular. Además, se calcula el flujo superficial por exceso de infiltración, fex, como la diferencia entre la precipitación y $f$. La evapotranspiración, $E a$, es calculada como una función de la evapotranspiración potencial y el almacenamiento de humedad de la zona radicular. Cuando la capacidad de la zona radicular es superada, todo el flujo pasa al compartimiento de la zona no saturada. Si el contenido de humedad de la zona no saturada es mayor al déficit de la zona saturada, se determina el exceso y este permite calcular el flujo superficial por saturación, $q 0$. Además, si hay déficit de la zona saturada, se genera un flujo vertical hacia el compartimiento de dicha zona, que depende de la profundidad del nivel freático y que a lo sumo llega a ser el contenido de humedad del compartimiento de la zona no saturada. Finalmente, se calcula el flujo en la zona saturada, $q s$.

En TOPMODEL se hace uso del Índice Topográfico de Kirkby (altan $\beta$ ) para identificar los puntos en la cuenca capaces de desarrollar condiciones de saturación y definir las áreas de contribución. Las suposiciones básicas, subyacentes al índice, en las que se basa el modelo son: 1)

a)

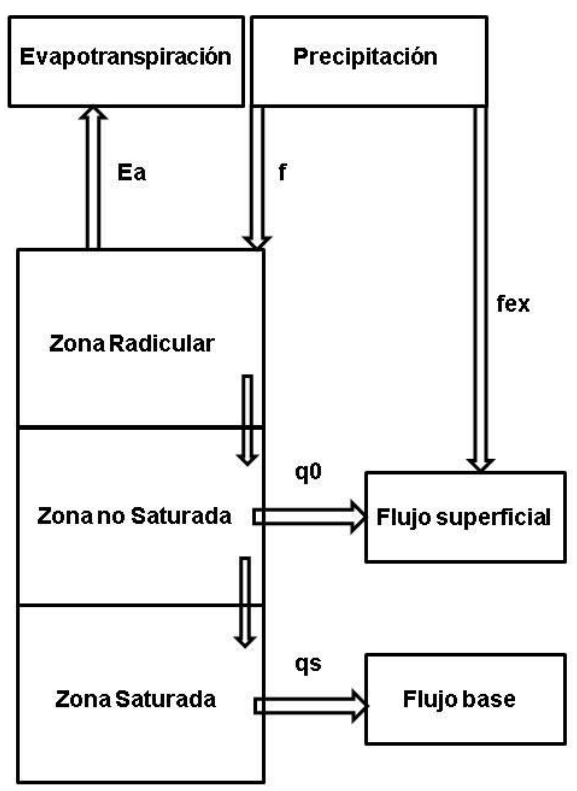

La dinámica de la zona saturada puede ser aproximada por representaciones de estado estable sucesivas de la zona saturada en un área $a$ drenando a un punto en la ladera. 2) El gradiente hidráulico de la zona saturada puede ser aproximado por la pendiente topográfica de la superficie local, $\tan \beta$. Una suposición adicional, el perfil de transmisividad puede ser descrito por una función exponencial del déficit de almacenamiento, $D_{i}$, con un valor de $T_{0}$ cuando el suelo está saturado en la superficie (Beven, 2012). Bajo estas suposiciones es descrito qs, el mismo $q s_{i}$ de las ecuaciones 1 y 2 (donde $m$ es un parámetro que controla la tasa de disminución de la transmisividad con incremento del déficit de almacenamiento y $r$ una tasa de recarga espacialmente homogénea). Además, las suposiciones permiten verificar la relación entre la profundidad del nivel freático local y el índice topográfico en ese punto. El cálculo requerido por TOPMODEL para generar un patrón de nivel freático local espacialmente distribuido se reduce a un cálculo para cada clase de índice topográfico.

$$
\begin{aligned}
q s_{i} & =T_{0} \tan \beta \exp \left(-D_{i} / m\right) \\
q s_{i} & =r a
\end{aligned}
$$

Finalmente, $q s$ permite determinar el flujo base. Mientras que los flujos fex y $q 0$ constituyen el flujo superficial.

Según la literatura, los conceptos de TOPMODEL no son aplicables en todas partes, debido a que los supuestos básicos subyacentes al índice se rompen particularmente en cuencas sujetas a una fuerte estación seca (Beven, 2012). A pesar de esto y de la fuerte estacionalidad presente en el área de estudio, dichos supuestos no se cuestionan en este trabajo. En todo caso se está planteando la necesidad de una mejor representación del comportamiento dinámico de los caudales, que pudiera lograrse con la representación del comportamiento hidrológico de los afloramientos rocosos. Según Rodríguez et al. (2014) el comportamiento dinámico

b)

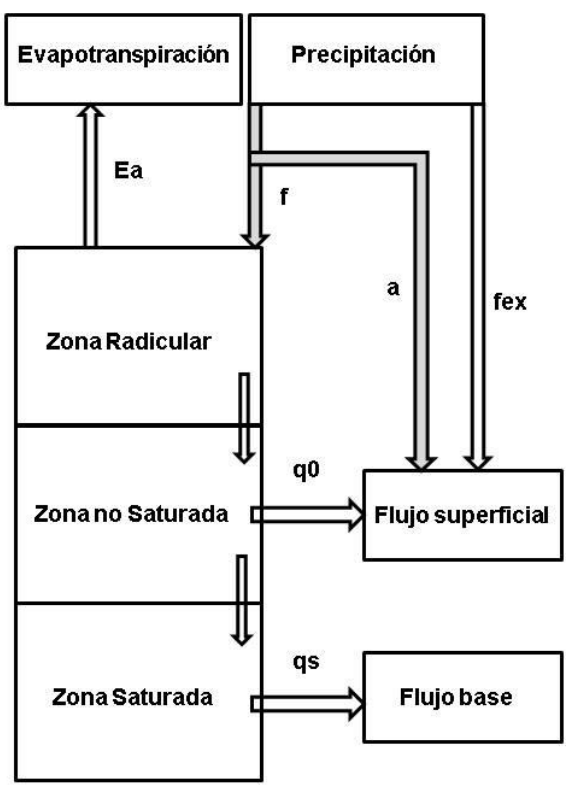

Figura 2. a) Estructura y flujos de TOPMODEL; b) Estructura y flujos de TOPMODEL modificado. 
(fuertemente dependiente de las precipitaciones en la época húmeda) debe estar asociado a la gran superficie de afloramientos rocosos, unidad sobre la que escurre la totalidad de las precipitaciones que recibe; y por otra parte a las laderas que son incapaces de retener gran parte del agua, al menos en los primeros $10 \mathrm{~cm}$ de profundidad. Las laderas podrían estar sujetas entonces a procesos de infiltración y rápida escorrentía subsuperficial (Rodríguez, 2010; Córdova, 2014). Sin embargo, durante la época seca se podría presumir un menor efecto de los afloramientos rocosos. Ya que el caudal no es tan sensible a las precipitaciones, pudiendo ser el resultado de bajos potenciales matriciales en los suelos, que retienen una gran parte de las pocas precipitaciones que ingresan y generan bajo drenaje (Rodríguez et al., 2014).

Por otro lado, la conectividad hídrica existente entre las laderas, lagunas y humedales contribuye a la recarga lenta y constante de las unidades de fondo de valle, cuyos suelos son los que mayor capacidad de almacenamiento de agua ofrecen, gracias a su alto contenido de materia orgánica (Córdova, 2014). Adicionalmente, los suelos orgánicos de los humedales mantienen elevados contenidos de agua permanentemente (Rodríguez et al., 2014). Estas unidades de fondo de valle, las zonas donde se ubican los humedales y las lagunas han de ser consideradas por el índice topográfico como propensas a saturación y el resto como menos propensas. La mayoría de los afloramientos rocosos han de ser etiquetados como menos propensos a saturación por su ubicación, y al considerarlos de esta forma o inclusive como propensos a saturación se modelaría un comportamiento que no se corresponde con el que estas unidades geomorfológicas realmente exhiben. Por lo tanto, la modificación de la estructura de TOPMODEL que se propone (Fig. 2b) consiste en redireccionar a flujo de afloramiento, $a$, una proporción del flujo que infiltraría.

\subsection{Datos disponibles y preprocesamiento}

Se dispuso de un modelo digital de elevaciones y una serie de datos de precipitación, caudal y evapotranspiración, provenientes de un sistema de monitoreo que fue implementado en el área de estudio en el año 2008 (Rodríguez, 2010).

El modelo digital de elevaciones usado tiene una resolución de $5 \mathrm{~m}$, este requirió la eliminación de sumideros, razón por la que se usó la función sinkfill del paquete TOPMODEL.

Los datos de caudal se estimaron a partir de mediciones hechas cada 10 minutos en un vertedero triangular $\left(90^{\circ}\right)$, ubicado en el punto de salida del valle. Se identifican dos grandes discontinuidades de datos de caudal antes de abril de 2011, razón por la que se define el periodo 01/09/2011$31 / 03 / 2012$ para calibración y del 01/09/2012 al 31/03/2013 para validación. Además, porque dichos periodos se corresponden a los mismos siete meses simulados en Yeguez et al. (2018), que incluyen meses húmedos, secos y de transición. La serie para calibración se caracteriza por caudal mínimo de 1e-07 m/10 min ${ }^{1}$, caudal máximo de $5.51 \mathrm{e}-05 \mathrm{~m} / 10 \mathrm{~min}$ y caudal medio de 5.61e-06 m/10 min. Mientras que para la serie de

${ }^{1}$ TOPMODEL requiere la altura del agua de lluvia, de evapotranspiración y caudal en m por paso de tiempo. Los datos de lluvia y evapotranspiración fueron convertidos de $\mathrm{mm}$ a $\mathrm{m}$. Los datos de caudal fueron convertidos $\mathrm{de}^{3} / \mathrm{s} \mathrm{a} \mathrm{m}^{3} / 10 \mathrm{~min}$ validación los estadísticos fueron $2 \mathrm{e}-07 \mathrm{~m} / 10 \mathrm{~min}, 4.48 \mathrm{e}-$ $05 \mathrm{~m} / 10 \mathrm{~min}$ y $4.07 \mathrm{e}-06 \mathrm{~m} / 10 \mathrm{~min}$, respectivamente.

Los datos de precipitación corresponden a mediciones hechas cada 10 minutos por una estación climatológica automatizada (resolución mínima $0.2 \mathrm{~mm}$ ), instalada en el fondo de valle del área de estudio. La serie para calibración se caracteriza por precipitación media de $1.51 \mathrm{e}-05 \mathrm{~m} / 10 \mathrm{~min}$ y precipitación máxima de $3.14 \mathrm{e}-03$ $\mathrm{m} / 10 \mathrm{~min}$. En validación la media fue $8.40 \mathrm{e}-06 \mathrm{~m} / 10 \mathrm{~min}$ y la máxima $3.1 \mathrm{e}-03 \mathrm{~m} / 10 \mathrm{~min}$.

Los datos de precipitación y caudal fueron almacenados en archivos de texto para ser leídos en R, como una serie de datos asociada a una secuencia temporal de 10 minutos. Lo que requirió identificar los datos faltantes, un $11 \%$ en la serie de caudal para validación. Estos fueron completados mediante interpolación lineal. Respecto a las series de precipitación no se identificó discontinuidades.

Los datos de evapotranspiración usados son estimaciones diarias de evapotranspiración de la vegetación ajustada a condiciones de estrés hídrico, calculada con el método de la FAO. Se prefiere esta estimación sobre la evapotranspiración de referencia, ya que esta última sobreestima en gran medida las salidas por este flujo, principalmente durante la época de sequía (Rodríguez, 2010). Cada estimación diaria se distribuyó en 144 valores, uno por cada 10 minutos, por medio de una distribución normal, imitando la función approx.pe.ts del paquete DYNATOPMODEL de R (Metcalfe, Beven, \& Freer, 2016).

\subsection{Calibración y validación}

Como en general no es posible estimar todos los parámetros de un modelo por mediciones directas o estimaciones previas, se usó para la calibración la simulación de Monte Carlo, que implica múltiples ejecuciones del modelo usando diferentes conjuntos de valores de los parámetros escogidos al azar (Beven, 2012). Algunos de estos parámetros se asocian a condiciones iniciales, tal como sucede en Buytaert \& Beven (2011) y Gil \& Tobón (2016).

Con la finalidad de poder establecer comparaciones se realizaron dos calibraciones, una antes y otra después de la modificación del modelo, usando la función topmodel del paquete TOPMODEL. Para cada calibración se realizaron 10,000 ejecuciones del modelo, usando las series de datos correspondientes al periodo 01/09/2011$31 / 03 / 2012$ y comenzando con los rangos de valores para cada parámetro propuestos por Buytaert \& Beven (2011), que también fueron usados por Gil \& Tobón (2016). Las descripciones de los nueve parámetros calibrados ( $q s 0$, $\operatorname{lnTe}, m, S r 0, S r m a x, t d, v r, k 0, C D)$ y los rangos finales de calibración para ambos casos se muestran en la Tabla 1.

Los rangos finales de calibración se obtuvieron ajustando los rangos sin afectar el desempeño de los modelos. Con cada ejecución de la función topmodel se obtuvo una matriz de descargas simuladas (con cada columna representando las descargas asociadas a un conjunto de parámetros) y un arreglo de eficiencias de Nash-Sutcliffe, una eficiencia para cada conjunto de parámetros, lo que

multiplicando por $600 \mathrm{seg}$. Luego los $\mathrm{m}^{3} / 10$ min se dividieron por el área de la cuenca en $\mathrm{m}^{2}$, para obtener el caudal en $\mathrm{m} / 10$ $\min$ 
Tabla 1. Los parámetros correspondientes a la mejor eficiencia obtenida en la calibración de TOPMODEL y TOPMODEL modificado y el rango final ${ }^{\text {a. }}$.

\begin{tabular}{|c|c|c|c|c|c|c|c|}
\hline \multirow{2}{*}{ Symbol } & \multirow{2}{*}{ Parámetro } & \multirow{2}{*}{ Unidad } & \multirow{2}{*}{ Min } & \multirow{2}{*}{$\operatorname{Max}$} & \multirow{2}{*}{$\begin{array}{l}\text { TOPMODEL } \\
\text { (calibración) }\end{array}$} & \multicolumn{2}{|c|}{ TOPMODEL modificado } \\
\hline & & & & & & $\mathrm{b}$ (calibrac.) & c (calibrac.) \\
\hline qs0 & $\begin{array}{l}\text { Flujo subsuperficial } \\
\text { inicial }\end{array}$ & $\mathrm{m}$ & $2 e-05$ & $6 e-05$ & $2.7 \mathrm{e}-05$ & $5.9 \mathrm{e}-05$ & $1.16 \mathrm{e}-04$ \\
\hline $\ln \mathrm{Te}$ & $\begin{array}{l}\text { Transmisividad (log } \\
\text { transformada) }\end{array}$ & $\mathrm{m}^{2} \mathrm{~h}^{-1}$ & 0 & 3 & 0.54 & 2.58 & 2.78 \\
\hline M & $\begin{array}{l}\text { Forma de la curva de } \\
\text { transmisividad }\end{array}$ & $\mathrm{m}$ & 0 & 0.06 & 0.03 & 0.02 & 0.02 \\
\hline Sr0 & $\begin{array}{l}\text { Déficit inicial de } \\
\text { almacenamiento de la } \\
\text { zona radicular }\end{array}$ & $\mathrm{m}$ & 0 & 0.05 & $7.85 e-03$ & 0.05 & 0.06 \\
\hline Srmax & $\begin{array}{l}\text { Máximo déficit de } \\
\text { almacenamiento de la } \\
\text { zona radicular }\end{array}$ & $\mathrm{m}$ & 0.05 & 0.2 & 0.10 & 0.17 & 0.20 \\
\hline $\mathrm{Td}$ & $\begin{array}{l}\text { Retraso de tiempo de la } \\
\text { zona no saturada }\end{array}$ & $\mathrm{h} \mathrm{m}^{-1}$ & 8 & 10 & 8.70 & 8.13 & 9.06 \\
\hline $\mathrm{vch}^{\mathrm{d}}$ & $\begin{array}{l}\text { Velocidad de flujo } \\
\text { terrestre }\end{array}$ & $\mathrm{m} \mathrm{h}^{-1}$ & 1000 & 1000 & 1000 & 1000 & 1000 \\
\hline $\mathrm{Vr}$ & $\begin{array}{l}\text { Velocidad de flujo del } \\
\text { canal }\end{array}$ & $\mathrm{m} \mathrm{h}^{-1}$ & 200 & 250 & 202.42 & 206.60 & 205.77 \\
\hline K0 & $\begin{array}{l}\text { Conductividad } \\
\text { hidráulica superficial }\end{array}$ & $\mathrm{m} \mathrm{h}^{-1}$ & 0 & 1 & 0.83 & 0.98 & 0.22 \\
\hline $\mathrm{CD}$ & Impulso capilar & $\mathrm{m}$ & 0 & 5 & 4.05 & 3.90 & 1.13 \\
\hline Dt & Paso de tiempo & $\mathrm{h}$ & 0.16 & 0.16 & 0.16 & 0.16 & 0.16 \\
\hline $\mathrm{E}$ & Eficiencia & & & & 0.624 & 0.716 & 0.756 \\
\hline
\end{tabular}

Leyenda;

a 10,000 iteraciones del modelo durante el proceso de calibración, usando el periodo 01/09/2011-31/03/2012

b Con parámetro $a=0.05$

${ }^{\mathrm{c}}$ Con parámetro $a=0.05$, la ampliación del límite superior de $q s 0$ y de $S r O$, y el aumento del límite inferior de $\operatorname{Srmax}$

d parámetro requerido, pero no es usado

permitió evaluarlos, así como también evaluar los modelos. La eficiencia de modelado de Nash-Sutcliffe es una medida cuantitativa de bondad de ajuste, también conocida como función objetivo o medida de rendimiento, ampliamente utilizada, basada en la varianza del error, que alcanza el valor de 1 para un ajuste perfecto (Beven, 2012).

Adicionalmente, se usó el método GLUE (por sus siglas en inglés, Generalized Likelihood Uncertainty Estimation) para generar límites de incertidumbre por medio del cálculo de cuantiles ponderados a partir de las predicciones de una batería de conjuntos de parámetros comportamentales. Ya que algunas veces un modelo puede lograr eficiencias Nash-Sutcliffe razonables, pero ser incapaz de generar límites de incertidumbre del $90 \%$ usando conjuntos de parámetros razonables (Buytaert \& Beven, 2011).

Para la validación de los modelos TOPMODEL y TOPMODEL modificado se usaron pruebas de registro dividido, tal como hicieron Buytaert \& Beven ( 2011) y Gil \& Tobón (2016). Una prueba de registro dividido consiste en hacer que el modelo use parámetros estimados en un primer periodo para predecir un segundo periodo, que en este caso corresponde a uno de la misma longitud del periodo de calibración, solo que un año después (01/09/2012-31/03/2013).

\section{RESULTADOS Y DISCUSIONES}

Usando la función topidx de TOPMODEL se calculó el índice topográfico (ver la Fig. 3a). Se puede apreciar que predominan los valores bajos. Mientras que los altos se localizan en fondo de valle, que son las zonas más propensas a saturación, donde se encuentra la red de drenaje, los humedales y las lagunas. Ya que las unidades de afloramientos rocosos se encuentran distribuidas a lo largo del área de estudio, estas son caracterizadas con valores de índice en todo su rango, tal como muestra el gráfico de barras de la Figura 3b, generado a partir del cruce de un mapa de afloramientos rocosos y el mapa del índice topográfico.

A pesar de que los afloramientos rocosos deberían permitir un escurrimiento de toda el agua que cae sobre ellos, a la mayoría se les asigna valores bajos del índice; es decir, se consideran zonas no propensas a la saturación, generándose flujo subsuperficial qué si bien sería menor al que se produciría en un área saturada, pudiera ser la razón por la que se tiene sobreestimación del flujo base.

Para las calibraciones, la función topmodel requirió además de las series de precipitación, caudal y evapotranspiración, una matriz con datos de las clases del índice topográfico (que se generó con la función make.classes del paquete TOPMODEL) y la función de retardo (que se generó con la función build_routing_table del paquete DYNATOPMODEL de R).

La calibración de TOPMODEL se puede ver en la Tabla 1 , donde se presenta el conjunto de parámetros correspondiente a la mejor eficiencia obtenida y el rango final de estos. La mayoría de los rangos de los parámetros mantuvieron en los valores iniciales o se acotaron luego de analizar los resultados de corridas preliminares. En el caso del parámetro Srmax se baja el límite inferior y para $t d$ se define un nuevo rango. 
a)

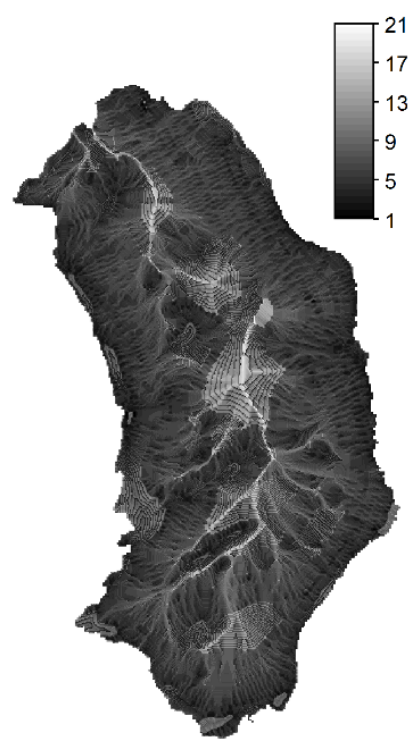

b)

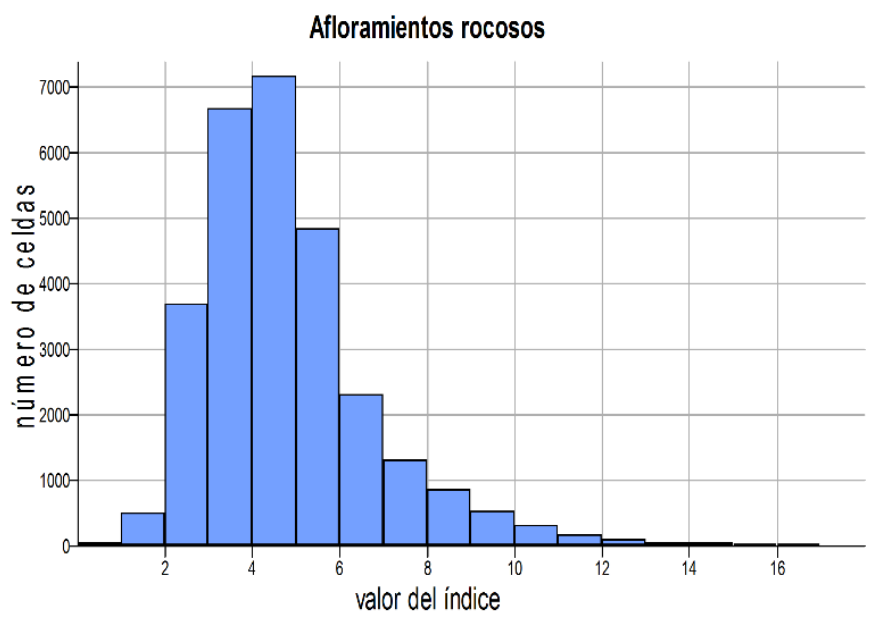

Figura 3. a) Índice topográfico en el área de estudio; b) Valores de índice asignados a los afloramientos rocosos.

Se observó que TOPMODEL no estaba simulando la totalidad de los eventos de precipitación debido a altos valores de Srmax, de este parámetro depende que el agua en la zona radicular pase o no al drenaje de la zona no saturada. Srmax está asociado a la capacidad de campo (Beven \& Kirkby, 1979), citando a Rodríguez (2010) el porcentaje de carbono orgánico presente en los suelos de Venezuela es menor al de los suelos de Ecuador y Colombia. Además, se han reportado correlaciones altamente significativas entre la cantidad de carbono orgánico en el suelo y su capacidad de campo.

La disminución del límite inferior del parámetro Srmax, plenamente justificada, implicó la diminución del límite superior de $\mathrm{Sr} 0$, ya que este último parámetro se encuentra supeditado por el primero. Estos cambios mejoraron la representación de eventos de precipitación y las eficiencias en consecuencia, pero trajo consigo una considerable disminución de los flujos de evapotranspiración simulados, ya que son precisamente estos parámetros los que participan en la determinación de la evapotranspiración.

Por otro lado, en los primeros intentos de modelado de una cuenca de páramo ecuatoriana se reportó insensibilidad del parámetro $t d$, razón por la que se eliminó argumentando que de esa forma se simplifica el modelo, quedando la zona no saturada y saturada unidas. En los suelos orgánicos del páramo es difícil distinguir el borde entre la zona saturada y la no saturada debido a las propiedades de expansión y contracción de los suelos, y la alta capacidad de almacenamiento de agua en condiciones insaturadas (Buytaert \& Beven, 2009). El parámetro también resultó insensible en el modelado de una cuenca colombiana (Gil \& Tobón, 2016). Según Güntner, Uhlenbrook, Seibert, \& Leibundgut (1999) $t d$ es parte de una formulación muy simple del movimiento vertical del agua del suelo y no tiene contrapartida física directa. Sin embargo, un valor alto de $t d$ se justifica si se tiene un valor alto de $T O$ (gran permeabilidad de los suelos) para que se produzca algún retraso en el movimiento del flujo entre la zona no saturada y la saturada (Oberto, 2006). Esto se observó en dos cuencas mediterráneas, con condición seca, donde los valores calibrados fueron 10.32 y 10.4 respectivamente (Piñol, Beven, \& Freer, 1997).

Un valor alto para $t d$, así como un valor bajo de $v r$ propician un retardo del flujo. En una cuenca ecuatoriana el valor calibrado de $v r$ fue $750 \mathrm{~m} / \mathrm{h}$, muy lento comparado con las estimaciones de campo. Lo que se atribuyó a la falta de un tercer reservorio fuertemente relacionado con grietas y macroporos (Buytaert, Célleri, De Bièvre, Deckers, \& Wyseure, 2004). En el caso del páramo venezolano, se presumiría entonces mayor necesidad de un tercer reservorio, ya que los valores calibrados de $v r$ son mucho más bajos. Otra posible explicación para este comportamiento podría ser la presunción de estado estacionario de la zona saturada, sobre la que se fundamenta TOPMODEL. El retraso sería un mecanismo usado por el modelo para sincronizar lo observado y simulado.

En diferentes corridas de calibración los valores de los mejores conjuntos de parámetros fueron muy similares, salvo el caso de $k 0$ y $C D$. Dichos parámetros son usados en la rutina de infiltración, y a partir del flujo que infiltra se determina el flujo sobre la tierra por exceso de infiltración. Por lo tanto, ambos flujos han de ser variables en las diferentes corridas; siendo muy bajo el flujo sobre la tierra por exceso de infiltración, en todos los casos.

La representación del comportamiento hidrológico de los afloramientos rocosos en TOPMODEL requirió redireccionar un porcentaje del flujo que infiltra a flujo superficial. Por lo tanto, se agregó un parámetro adicional, $a$, la proporción de flujo de precipitación a redireccionar. Dicho parámetro se fijó inicialmente en 0.20 , por ser aproximadamente el área que cubren los afloramientos rocosos, pero al observar un bajo desempeño del modelo se decidió calibrarlo también. Las mejores eficiencias fueron obtenidas usando valores de $a$ entre 0.04 y 0.05 inclusive.

En la Tabla 1 se muestra la calibración del TOPMODEL modificado usando $a=0.05$, específicamente la eficiencia obtenida y los valores del resto de parámetros (manteniendo los rangos usados para la calibración de TOPMODEL). Se puede observar que la modificación 
hecha al modelo mejora la eficiencia en calibración. Mejores resultados se obtuvieron ampliando el límite superior de $q s O$ y de $\mathrm{SrO}$ (condiciones iniciales), con el respectivo aumento del límite inferior de Srmax. Al conservar los rangos o al ampliarlos, los mejores valores de los parámetros se mantuvieron muy similares en las diferentes corridas, con las excepciones $k O$ y $C D$, como sucedió con TOPMODEL.

La Figura 4 muestra el caudal observado y el simulado por TOPMODEL y TOPMODEL modificado luego de la calibración. Se observa que TOPMODEL modificado hace en general una mejor representación de los picos y las curvas de recesión, aun cuando estas últimas siguen sobreestimadas están la mayor parte del tiempo más próximas a los datos observados que sus pares de TOPMODEL.

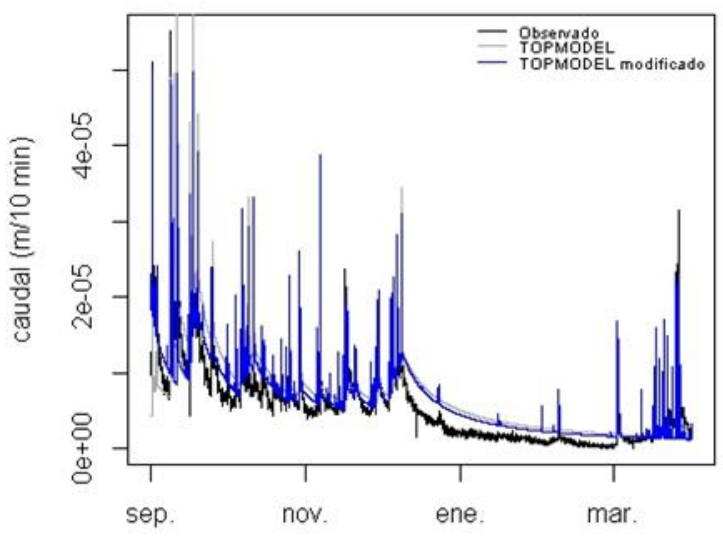

Figura 4. Caudal observado y el simulado por TOPMODEL y TOPMODEL modificado en calibración.

El valor calibrado de $a$ resultó mucho menor a 0.20 , quizá porque solo los afloramientos rocosos próximos a la red de drenaje contribuyen a la escorrentía rápida. Afloramientos ubicados a lo sumo a $44 \mathrm{~m}$ de la red de drenaje constituyen un $25 \%$ del área de afloramientos, de acuerdo con el cálculo de las distancias entre los diferentes elementos topográficos y la red de drenaje hecho con ILWI $^{2}$. Por lo tanto, si consideramos que la precipitación se distribuye de forma uniforme en la zona de estudio, un $5 \%$ de ella caería sobre los afloramientos ubicados a lo sumo a $44 \mathrm{~m}$ de la red de drenaje, porcentaje de flujo que se redireccionó en la versión mostrada de TOPMODEL modificado. Sin embargo, otros factores podrían explicar el bajo valor de $a$, como la presencia de fracturas en los afloramientos y el efecto de la fuerte evapotranspiración.

Se determinaron los límites de incertidumbre del $95 \%$ para TOPMODEL y TOPMODEL modificado, descartando los conjuntos de parámetros con eficiencia menor a 0.3 . Más que comparar la estrechez de los límites, se notó que el caudal observado se encuentra fuera de los límites por más tiempo en el caso de TOPMODEL que en el caso de TOPMODEL modificado. Se calcularon los límites del $95 \%$ y no los de $90 \%$, ya que es más fácil apreciar que el caudal observado y el límite inferior de TOPMODEL modificado coinciden la mayor parte del tiempo. Según este criterio TOPMODEL modificado es una mejor representación.

${ }^{2}$ El Sistema Integrado de Información de Tierras y Aguas (ILWIS: Integrated Land and Water Information system) es un

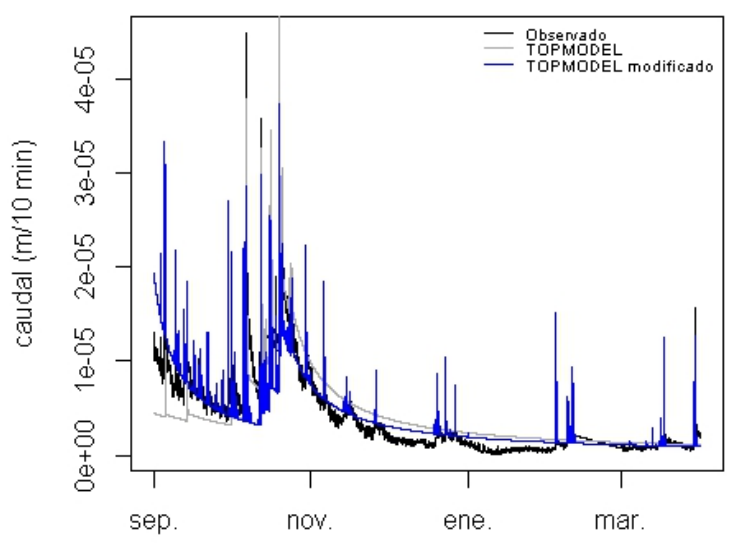

Figura 5. Caudal observado y el simulado por TOPMODEL y TOPMODEL modificado en validación.

La validación de los modelos se llevó a cabo usando los parámetros obtenidos de sus mejores calibraciones y las series de precipitación y evapotranspiración del 01/09/2012 al 31/03/2013. La Figura 5 muestra el caudal observado y el simulado por TOPMODEL y TOPMODEL modificado en validación. La eficiencia obtenida por TOPMODEL fue de 0.72 y la obtenida por TOPMODEL modificado de 0.76 , eficiencias mejores o iguales a las obtenidas en calibración respectivamente. TOPMODEL modificado obtiene solo una pequeña ventaja quizá por tratarse de un año más seco con un porcentaje de datos interpolados; sin embargo, imita mejor la dinámica que su contraparte, que genera curvas de recesión generalmente más suaves.

\section{CONCLUSIONES}

Se modificó TOPMODEL buscando representar el comportamiento hidrológico de los afloramientos rocosos presentes en cuencas de páramo andino venezolano, dicha modificación se llevó a cabo considerando el conocimiento obtenido en investigaciones previas. El TOPMODEL modificado parece representar mejor la dinámica que TOPMODEL, mejoró los picos y las curvas de recesión, obteniendo eficiencias Nash-Sutcliffe superiores tanto en calibración como en validación. Además, logra contener la descarga observada dentro de límites de incertidumbre del $95 \%$ por más tiempo. Asumiendo un coeficiente de escorrentía de 1 en los afloramientos y de acuerdo con la calibración, la lluvia que cae en un $25 \%$ del área de afloramientos es redireccionada a escorrentía superficial, posiblemente se trate del conjunto de afloramientos ubicados a lo sumo a $44 \mathrm{~m}$ de la red de drenaje. Sin embargo, otras interpretaciones son posibles ya que otros factores podrían explicar el bajo valor de $a$, como la presencia de fracturas y el efecto de la fuerte evapotranspiración. Por lo tanto, sería conveniente realizar más estudios sobre la respuesta hidrológica de los afloramientos rocosos presentes en el área de estudio a diferentes escalas; así como también la

sistema de información geográfica (SIG) y software de detección remota para el procesamiento de vectores y ráster. 
evaluación de diferentes hipótesis mediante el uso de modelos.

Por otro lado, la mayoría de los valores calibrados de los parámetros, tanto para el caso de TOPMODEL como de TOPMODEL modificado, pertenecen a un rango con significado físico. Algunos ajustes debieron hacerse en un par de parámetros en función de las características particulares del páramo andino venezolano. Destaca el comportamiento de $v r$ que parece estar revelando deficiencias de los modelos para la representación. Otros aspectos que requieren de una evaluación es el uso del modelo Green y Ampt en la determinación del flujo para infiltración y la presunción de estado estacionario de la zona saturada, sobre la que se fundamenta TOPMODEL.

\section{REFERENCIAS}

Beven, K. (2012). Rainfall-Runoff Modelling: The Premier (2 ${ }^{\mathrm{a}}$ ed.). Wiley-Blackwell.

Beven, K., \& Kirkby, M. (1979). A physically based, variable contributing area model of basin hydrology. Hydrological Sciences Bulletin, 24(1), 43-69. doi:10.1016/j.scitotenv.2006.12.013

Burns, D. A., McDonnell, J. J., Hooper, R. P., Peters, N. E. Freer, J. E., Kendall, C., \& Beven, K. (2001). Quantifying contributions to storm runoff through endmember mixing analysis and hydrologic measurements at the Panola Mountain research watershed (Georgia, USA). Hydrological Processes, 15, 1903-1924.

Buytaert, W. (2009). Implementation of the hydrological model TOPMODEL in R. Disponible en https://cran.rproject.org/web/packages/topmodel/topmodel.pdf

Buytaert, W., \& Beven, K. (2009). Regionalization as a learning process. Water Resources Research, 45(11), 1-13. doi:10.1029/ 2008WR007359

Buytaert, W., \& Beven, K. (2011). Models as multiple working hypotheses: Hydrological simulation of tropical alpine wetlands. Hydrological Processes, 25, 1784-1799.

Buytaert, W., Célleri, R., De Bièvre, B., Deckers, J., \& Wyseure, G. (2004). Modelando el comportamiento hidrológico de microcuencas de páramo en el Sur del Ecuador con TOPMODEL. In: Memorias del III Congreso Latinoamericano sobre Manejo de Cuencas Hidrográficas. Arequipa.

Buytaert, W., Iñiguez, V., \& De Bièvre, B. (2007). The effects of afforestation and cultivation on water yield in the Andean páramo. Forest Ecology and Management, 251(1-2), 22-30.

Córdova, C. (2014). Estudio de las características edáficas con el fin de estimar la capacidad potencial de almacenamiento hídrico en los suelos minerales de la Microcuenca de Miguaguó, Mixteque.Tesis de Postgrado, 102 pp.Universidad de los Andes, Mérida, Venezuela.

Devi, G. K., Ganasri, P. B., \& Dwarakish, G. S. (2015). A review on hydrological models. Aquatic Procedia, 4 , 1001-1007.

https://doi.org/10.1016/j.aqpro.2015.02.126

Durand, P., Robson, A., \& Neal, C. (1992). Modelling the hydrology of submediterranean montane catchments (Mont-Lozère, France) using TOPMODEL: initial results. Journal of Hydrology, 139, 1-14. https://doi.org/10.1016/00221694(92)90191-W

FAO. (2009). Guía para la descripción de suelos. Roma. Disponible en http://www.fao.org/3/a-a0541s.pdf

Gil, E., \& Tobón, C. (2016). Hydrological modelling with TOPMODEL of Chingaza páramo, Colombia. Revista Facultad Nacional de Agronomía, 69(2), 1-16.

Güntner, A., Uhlenbrook, S., Seibert, J., \& Leibundgut, C. (1999). Multi-criterial validation of TOPMODEL in a mountainous catchment. Hydrological Processes, 13, 1603-1620.

Wilson, J. P., \& Gallant, J. C. (2000). Terrain Analysis: Principles and Applications. John Wiley \& Sons, Inc.

Lanni, C., McDonnell, J. J., \& Rigon, R. (2011). On the relative role of upslope and downslope topography for describing water fl ow path and storage dynamics : a theoretical analysis. Hydrological Processes, 25, 39093923.

Metcalfe, P., Beven, K., \& Freer, J. (2016). Implementation of the dynamic "TOPMODEL" hydrological model. Disponible en https://cran.rproject.org/web/packages/dynatopmodel/dynatopmodel .pdf

Oberto, L. (2006). Evaluación de la Aplicación del Modelo Lluvia- escorrentía TOPMODEL a la cuenca del Embalse Yacambú. Tesis de Postgrado. 69 pp.Universidad Centrooccidental Lisandro Alvarado, Barquisismeto, Venezuela.

OMM, \& UNESCO. (2012). Glossario hidrológico internacional. Junta de publicaciones Organización Meteorológica Mundial (OMM).

Piñol, J., Beven, K., \& Freer, J. (1997). Modelling the hydrological response of mediterranean catchments, Prades, Catalonia. The use of distributed models as aids to hypothesis formulation. Hydrological Processes, 11, 1287-1306. https://doi.org/10.1002/(SICI)1099-1085(199707)11

Rodríguez, M. (2010). El páramo como proveedor de agua: análisis de las unidades geomorfológicas y de vegetación sobre el balance hídrico de una microcuenca andina de venezuela. Tesis de Postgrado, 140 pp.Universidad de los Andes. Mérida, Venezuela.

Rodríguez, M., Acevedo, D., Buytaert, W., Ablan, M., \& De Bièvre, B. (2014). El páramo andino como productor y regulador del recurso agua. El caso de la microcuenca alta de la Quebrada Mixteque, Sierra Nevada de Mérida, Venezuela. En: Cuesta F, Sevink J, Llambí LD, De Bièvre B, Posner J, Editores.Avances en investigación paara la conservación de los páramos andinos, CONDESAN, 245-266.

Seibert, J., Bishop, K. H., \& Nyberg, L. (1997). A test of Topmodel's ability to predict spatially distributed groundwater levels. Hydrological Processes, 11, 11311144.

Suarez, V. V. C., Okello, A. M. L. S., Wenninger, J. W., \& Uhlenbrook, S. (2015). Understanding runoff processes in a semi-arid environment through isotope and hydrochemical hydrograph separations. Hydrology and Earth System Sciences, 19, 4183-4199. doi:10.5194/hess-19-4183-2015 
Tarolli, P., Borga, M., \& Fontana, G. D. (2008). Analysing the influence of upslope bedrock outcrops on shallow landsliding. Geomorphology, 93, 186-200. https://doi.org/10.1016/j.geomorph.2007.02.017

Woo, M. (2012). Permafrost Hydrology. Springer Verlag, Berlin.
Yeguez, M., Ablan, M., Acevedo, D., Rodríguez, M., \& Machado, D. (2018). Primer intento de modelización de la hidrología de una microcuenca del páramo andino venezolano. In: VI Jornadas del Liancol y II Congreso de Ciencias Ambientales, Cabimas, Venezuela, pp. 104-109. 